\title{
2-Ring Heterocyclic Compound
}

National Cancer Institute

\section{Source}

National Cancer Institute. 2-Ring Heterocyclic Compound. NCI Thesaurus. Code C543.

An organic compound composed of two ring structures, where at least one ring contains

more than one kind of atom. 